\title{
O GÊNERO TRACHELOMONAS (EUGLENOPHYTA) DE DUAS ÁREAS DE PROTEÇÃO AMBIENTAL, APA RIO CAPIVARA E APA LAGOAS DE GUARAJUBA, MUNICÍPIO DE CAMAÇARI, BAHIA, BRASIL.
}

\author{
$\underline{\text { Adones de Jesus Santos Pereira }}{ }^{1}$; Carlos Wallace do Nascimento Moura ${ }^{2}$ \\ 1. Bolsista PIBIC/CNPQ, Graduando em Bacharelado em Ciências Biológicas, Universidade Estadual de Feira de \\ Santana, e-mail: adones2006@gmail.com \\ 2. Orientador, Departamento de Ciências Biológicas, Universidade Estadual de Feira de Santana, e-mail: \\ wallace@uefs.br
}

PALAVRAS-CHAVE: Microalga; Taxonomia; Inventário.

\section{INTRODUÇÃO}

Euglenophyta é uma das muitas divisões de microalgas existentes, englobando atualmente 40 gêneros e aproximadamente 800 espécies (Parra \& Bicudo, 1995), sendo Trachelomonas Ehrenberg emend. Deflandre o gênero mais representativo da divisão, com cerca de 370 táxons aceitos taxonomicamente (Guiry \& Guiry 2018).

O gênero inclui algas de vida livre e hábito solitário, caracterizadas por possuir célula com um envelope rígido e mucilaginoso denominado de lórica, apresentando um poro no polo anterior do qual emerge o flagelo. A lórica apresenta diferentes formatos e padrões de ornamentação, os quais são empregados na taxonomia do grupo (Tell \& Conforti, 1986), e uma coloração castanho-amarelada, ocasionada pela impregnação de compostos férricos e de manganês (Dunlap et al. 1983).

Trabalhos taxonômicos de microalgas no estado do Bahia ainda são escassos, diante da complexidade de suas bacias de drenagem e da diversidade de ecossistemas aquáticos continentais. Com relação ao gênero Trachelomonas, todas as informações disponíveis estão restritas aos seguintes trabalhos: Fuentes et al. 2010; Santana, 2011; Severiano et al. 2012 e Alves-da-Silva \& Menezes, 2015, embora os autores mencionados não forneçam descrição e ilustrações em seus trabalhos que auxiliassem a confirmação dos táxons, existem também dois estudos taxonômicos (Pereira, 2016; 2017), onde, ao todo foram identificados 39 táxons a nível infraespecífico.

Diante do que foi exposto, o presente trabalho teve como objetivo a realização de um estudo taxonômico das microalgas pertencentes ao gênero Trachelomonas ocorrentes em duas áreas de proteção ambiental, APA Rio Capivara e APA Lagoas de Guarajuba, município de Camaçari, Bahia, Brasil, de modo a ampliar o registro de espécies para a Bahia e, consequentemente, para o Brasil.

\section{MATERIAL E MÉTODOS}

Foram analisadas amostras tombadas na coleção líquida do Herbário da Universidade Estadual de Feira de Santana (HUEFS), coletadas em 2007, em duas áreas de Preservação Ambiental (APA): APA Rio Capivara e APA Lagoas de Guarajuba, ambas situadas no município de Camaçari, região Metropolitana de Salvador, Bahia.

Analisou-se ao todo 120 unidades amostrais, 60 coletadas no verão (janeiro-março de 2007) e 60 no inverno (junho-agosto de 2007), sendo 30 amostras para cada APA em cada período de amostragem.

As amostras foram obtidas com o auxílio de uma rede de plâncton (malha com $20 \mu \mathrm{m}$ de abertura) e através do espremido manual das raízes e folhas de plantas submersas. Após a coleta o material foi imediatamente fixado em solução de Transeau (Bicudo \& Menezes 2006). 
No laboratório, as amostras foram analisadas com auxílio do microscópio óptico binocular marca Olympus (modelo BX43) e as fotomicrografias obtidas com máquina fotográfica digital (Modelo QImaging 5.0) acoplado ao microscópio.

As dimensões dos espécimes foram aferidas através de ocular micrométrica e estão representadas pelos limites métricos mínimos e máximos. Aproximadamente 480 alíquotas foram preparadas das unidades amostrais e analisadas. A identificação taxonômica foi, sempre que possível, em nível infragenérico, analisando-se as características morfológicas e métricas das populações. Estas foram baseadas em trabalhos especializados, incluindo floras, revisões, dissertações e teses.

O sistema de classificação utilizado segue Guiry \& Guiry (2018).

\section{RESULTADOS E DISCUSSÃO}

Das 120 unidades amostrais analisadas, 67 apresentaram representantes de Trachelomonas, sendo 33 unidades na Apa Rio Capivara e 34 na APA Lagoas de Guarajuba.

O gênero Trachelomonas foi representado na flora local por 23 táxons, sendo 21 identificados a nível infraespecífico (17 espécies, oito variedades que não as típicas de suas respectivas espécies, uma forma taxonômica não típica, porém, pertencente à variedade típica de sua espécie [Trachelomonas armata var. armata f. inevoluta] e uma outra forma taxonômica não típica, pertencente a uma variedade igualmente não típica de sua espécie [Trachelomonas raciborskii var. nova f. minor], além de uma variedade não identificada pertencente a $T$. kellogii e um táxon identificado apenas a nível de gênero.

A APA Lagoas de Guarajuba apresentou maior riqueza taxonômica (21 táxons) quando comparado a APA Rio Capivara (17 táxons).

Dentre os 23 táxons identificados, 15 foram comuns às duas áreas (Tab. 1), seis foram exclusivos da APA Lagoas de Guarajuba (T. allia var. obesa, T. armata var. steinii, T. conica, T. curta var. curta, T. kellogii var., T. raciborskii var. nova f. minor) e dois exclusivos da APA Rio Capivara (T. crispa, Trachelomonas sp.) (Tab. 1).

O táxon mais representativo no presente estudo foi $T$. volvocinopsis var. volvocinopsis, registrado em 30 unidades amostrais das 67 onde houveram registros de Trachelomonas.

Ao comparar a ocorrência dos 23 táxons inventariados nas duas áreas de proteção ambiental (APA Lagoas de Guarajuba e APA Rio Capivara) nas estações amostradas (Verão e Inverno), constatou-se que três táxons foram exclusivos do verão (T. armata var. steinii, $T$. crispa e Trachelomonas sp.) e dois exclusivos do inverno (T. kellogii var. e T. raciborskii var. nova f. minor). Levando em consideração a exclusividade de cada área, dentro de cada período, a APA Rio Capivara apresentou cinco táxons exclusivos no verão e dois no inverno, enquanto que a APA Lagoas de Guarajuba teve quatro táxons exclusivos no verão e seis no inverno. A APA Rio Capivara apresentou maior riqueza de táxons no verão (15 táxons) quando comparado ao inverno (12 táxons), ao passo que a APA Lagoas de Guarajuba apresentou maior riqueza no inverno (17 táxons), quando comparado ao verão (15 táxons).

\section{CONCLUSÕES}

O estudo das amostras coletadas nas duas áreas de proteção ambiental, APA Rio Capivara e APA Lagoas de Guarajuba, município de Camaçari, Bahia, Brasil, permitiu concluir que: 1. A área apresentou expressiva biodiversidade, tendo sido registrados 23 táxons; 2. Três táxons de Trachelomonas constituem novos registros para a região Nordeste do Brasil e consequentemente para a Bahia (T. cervicula, T. crispa e T. lacustris var. ovalis); 3. Um táxon (Trachelomonas sp.) não pôde ser enquadrado em quaisquer circunscrição de Trachelomonas já descritas e, muito provavelmente, constitui-se como uma novidade para a ciência; 4. A APA Lagoas de Guarajuba apresentou maior riqueza taxonômica (21 táxons) 
quando comparado a APA Rio Capivara (17 táxons); 5. Dentre os táxons identificados, 15 foram comuns às duas áreas, seis foram exclusivos da APA Lagoas de Guarajuba e dois exclusivos da APA Rio Capivara; 6. Não houve diferença significativa entre a riqueza taxônomica registrada nas duas estações, com 21 táxons registrados no verão e 20 no inverno; 7. A APA Rio Capivara apresentou cinco táxons exclusivos no verão e dois no inverno, enquanto que a APA Lagoas de Guarajuba teve quatro táxons exclusivos no verão e seis no inverno; 8. A APA Rio Capivara apresentou maior riqueza de táxons no verão (15 táxons) quando comparado ao inverno (12 táxons), ao passo que a APA Lagoas de Guarajuba apresentou maior riqueza no inverno (17 táxons), quando comparado ao verão (15 táxons); 9. O táxon mais representativo do estudo foi $T$. volvocinopsis var. volvocinopsis, registrado em 30 unidades amostrais das 120 analisadas.

Tabela. 1. Listagem dos táxons de Trachelomonas (Euglenophyceae) anteriormente referidos para o Estado e dos dados obtidos nas duas áreas de proteção ambiental, APA Rio Capivara e APA Lagoas de Guarajuba, município de Camaçari, Bahia, Brasil.; Citações anteriores: 1- Fuentes et al. (2010), 2- Santana (2011), 3- Severiano et al. (2012), 4- Alves-da-Silva \& Menezes (2015), 5- Pereira (2016), 6 - Pereira (2017); Presente trabalho: NE- Novo registro para o Nordeste do Brasil, BA- novo registro para a Bahia, ARC $=$ ocorrência registrada na APA Rio Capivara, ALG = ocorrência registrada na APA Lagoas de Guarajuba.

\begin{tabular}{|c|c|c|c|c|c|}
\hline \multirow[b]{2}{*}{ Táxons } & \multirow{2}{*}{$\begin{array}{r}\text { Citações } \\
\text { anteriores }\end{array}$} & \multicolumn{3}{|c|}{ Presente trabalho } & \multirow[b]{2}{*}{ ALG } \\
\hline & & BA & NE & ARC & \\
\hline T. abrupta Swirenko emend. Deflandre var. abrupta & $2 ; 5 ; 6$ & & & $\mathrm{X}$ & $\mathrm{X}$ \\
\hline T. abrupta Swirenko emend. Deflandre var. arcuata (Playfair) Deflandre & $2 ; 5$ & & & & \\
\hline T. acanthophora Stokes & 2 & & & & \\
\hline T. acanthophora A.C.Stokes var. speciosa (Deflandre) Balech & 6 & & & & \\
\hline T. allia Drezepolski emend. Deflandre var. obesa Balech & $5 ; 6$ & & & & $\mathrm{X}$ \\
\hline T. armata (Ehrenberg) F.Stein var. armata f. armata & $2 ; 3 ; 5 ; 6$ & & & & \\
\hline T. armata (Ehrenberg) F.Stein var. armata f. inevoluta Deflandre & $5 ; 6$ & & & $\mathrm{X}$ & $\mathrm{X}$ \\
\hline T. armata (Ehrenberg) F.Stein var. litoralensis Tell \& Domitrovic & $2 ; 5 ; 6$ & & & & \\
\hline T. armata (Ehrenberg) F.Stein var. longispina Playfair emend. Deflandre & $2 ; 5 ; 6$ & & & $\mathrm{X}$ & $\mathrm{X}$ \\
\hline T. armata var. ovata Svirenko & 2 & & & & \\
\hline T. armata (Ehrenberg) F.Stein var. sparsigranosa Playfair & 5 & & & & \\
\hline T. armata (Ehrenberg) F.Stein var. steinii Lemmermann emend. Deflandre & $2 ; 5 ; 6$ & & & & $\mathrm{X}$ \\
\hline T. balechii Tell & 5 & & & $\mathrm{X}$ & $\mathrm{x}$ \\
\hline T. bulla F.Stein emend. Deflandre. var. bulla & 2 & & & & \\
\hline T. cervicula A.Stokes & & $\mathrm{x}$ & $\mathrm{x}$ & $\mathrm{X}$ & $\mathrm{X}$ \\
\hline T. conica Playfair & $5 ; 6$ & & & $\mathrm{x}$ & $\mathrm{X}$ \\
\hline T. crispa Balech & & $\mathrm{x}$ & $\mathrm{x}$ & $\mathrm{x}$ & \\
\hline T. curta A.M.Cunha emend. Deflandre var. curta & 5 & & & & $\mathrm{X}$ \\
\hline T. curta A.M.Cunha emend. Deflandre var. subpunctata Bourrelly & 5 & & & & \\
\hline T. dangeardiana (Deflandre) Huber-Pestalozzi var. glabra (Playfair) Deflandre & 5 & & & & \\
\hline T. dastuguei Balech var. dastuguei f. dastuguei & 2 & & & & \\
\hline T. hemispherica Garcia-de-Emiliani & 2 & & & & \\
\hline T. hirta A.M.Cunha var. hirta & 2 & & & & \\
\hline T. hispida (Perty) F.Stein emend. Deflandre var. hispida & $2 ; 5 ; 6$ & & & $\mathrm{x}$ & $\mathrm{x}$ \\
\hline T. hispida (Perty) F.Stein emend. Deflandre var. coronata Lemmermann & $5 ; 6$ & & & & \\
\hline T. hispida (Perty) F.Stein emend. Deflandre var. crenulatocollis (Maskell) & 6 & & & & \\
\hline T. hispida (Perty) F.Stein emend. Deflandre var. duplex Deflandre & $5 ; 6$ & & & $\mathrm{X}$ & $\mathrm{X}$ \\
\hline T. hispida (Perty) F.Stein emend. Deflandre var. punctata Lemmermann & 5 & & & & \\
\hline T. intermedia P.A.Dangeard var. intermedia & $5 ; 6$ & & & $\mathrm{X}$ & $\mathrm{X}$ \\
\hline T. intermedia P.A.Dangeard var. minor Tell & $5 ; 6$ & & & $\mathrm{X}$ & $\mathrm{X}$ \\
\hline T. kellogii Skvortzov emend. Deflandre var. kellogii & $5 ; 6$ & & & $\mathrm{x}$ & $\mathrm{X}$ \\
\hline T. kellogii var. & & & & & $\mathrm{X}$ \\
\hline T. lacustris Drezepolski var. lacustris & $2 ; 5 ; 6$ & & & & \\
\hline T. lacustris Drezepolski var. ovalis & & $\mathrm{x}$ & $\mathrm{x}$ & $\mathrm{X}$ & $\underline{X}$ \\
\hline T. lemmermannii Woloszýnska emend. Deflandre var. lemmermannii & $5 ; 6$ & & & & \\
\hline T. lemmermannii Woloszýnska emend. Deflandre var. acuminata Deflandre & $2 ; 6$ & & & $\mathrm{X}$ & $\mathrm{X}$ \\
\hline T. magdaleniana Deflandre & 6 & & & & \\
\hline T. megalacantha A.M.Cunha var. megalacantha & $5 ; 6$ & & & & \\
\hline T. obesa Ehrenberg & $1 ; 3$ & & & & \\
\hline T. oblonga Lemmermann var. oblonga & $1 ; 3 ; 5$ & & & & \\
\hline T. parvicollis Deflandre & 2 & & & & \\
\hline T. raciborskii Woloszýnska var. raciborskii & $2 ; 5$ & & & & \\
\hline T. raciborskii Woloszýnska var. nova Drezepolski & $5 ; 6$ & & & & \\
\hline T. raciborskii Woloszýnska var. nova Drezepolski f. minor T. Hortobágyi & 5 & & & & X \\
\hline T. robusta Svirenko var. robusta & $5 ; 6$ & & & $\mathrm{X}$ & $\mathrm{x}$ \\
\hline T. rugulosa F.Stein var. rugulosa & 5 & & & & \\
\hline T. spinosa A.C.Stokes & 6 & & & & \\
\hline T. sculpta Balech var. sculpta & 5 & & & & \\
\hline
\end{tabular}




\begin{tabular}{|c|c|c|c|c|c|}
\hline \multirow[b]{2}{*}{ Táxons } & \multirow{2}{*}{$\begin{array}{c}\text { Citações } \\
\text { anteriores }\end{array}$} & \multicolumn{4}{|c|}{ Presente trabalho } \\
\hline & & BA & $\mathbf{N E}$ & ARC & ALG \\
\hline T. similis Stokes & 2 & & & & \\
\hline T. superba Svirenko emend. Deflandre & $2 ; 5 ; 6$ & & & & \\
\hline T. volvocina (Ehrenberg) Ehrenberg var. volvocina & $1 ; 2 ; 3 ; 4 ; 5 ; 6$ & & & $\mathrm{X}$ & $\mathrm{X}$ \\
\hline T. volvocina Ehrenberg var. compressa Deflandre & 2 & & & & \\
\hline T. volvocinopsis Svirenko var. volvocinopsis & $5 ; 6$ & & & $\mathrm{X}$ & $\mathrm{x}$ \\
\hline Trachelomonas sp. & & & & $\mathrm{x}$ & \\
\hline
\end{tabular}

\section{REFERÊNCIAS:}

ALVES-DA-SILVA, S.M.; MENEZES, M. 2015. Euglenophyceae in Lista de Espécies da Flora do Brasil. Jardim Botânico do Rio de Janeiro. Disponivel em: <http://floradobrasil.jbrj.gov.br/jabot/floradobrasil/FB109138>

DUNLAP, J.R.; WALNE, P.L.; BENTLEY, J. 1983. Microarchitecture and elemental spatial segregation of envelopes of Trachelomonas lefevrei (Euglenophyceae). Protoplasma 117: 97-106

FUENTES, E.V.; OLIVEIRA, H.S.B.; CORDEIRO-ARAÚJO, M.K.; SEVERI, W.; MOURA, A.N. 2010. Variação espacial e temporal do fitoplâncton do Rio de Contas, Bahia, Brasil. Revista Brasileira de Engenharia de Pesca 5(2): 13-25

GUIRY IN GUIRY, M.D.; GUIRY, G.M. 2018. AlgaeBase. World-wide electronic publication, National University of Ireland, Galway. http://www.algaebase.org. Acesso em 15/07/2018.

PARRA, O.O.; BICUDO, C.E.M. 1995. Introdução a la biología y sistemática de las algas continentales. Chile:Universidad de Concepción.

PEREIRA, A.J.S. 2016. O gênero Trachelomonas (Euglenophyceae) de duas áreas dos Marimbus (Baiano e Remanso), Chapada Diamantina, Bahia, Brasil. Relatório de Inciação Científica - FAPESB - XX SEMIC - Universidade Estadual de Feira de Santana (UEFS).

PEREIRA, A.J.S. 2017. O gênero Trachelomonas (Euglenophyta) dos municípios de São Sebastião do Passé, Candeias, Mata de São João, Pojuca e São Francisco do Conde, Região Metropolitana de Salvador, Bahia, Brasil. Relatório de Iniciação Ciêntífica - FAPESB - XXI SEMIC - Universidade Estadual de Feira de Santana (UEFS).

SANTANA, L.M. 2011. Análise física e química da água e estrutura da comunidade fitoplanctônica do rio Almada (sul da Bahia). Dissertação de Mestrado, Programa de Pós-Graduação em Sistemas Aquáticos Tropicais, Universidade Estadual de Santa Cruz, Bahia.

SEVERIANO, J.S.; MOURA, A.N.; BEZERRA-OLIVEIRA, H.S.; CORDEIRO-ARAÚJO, M.K.; DANTAS, E.W. 2012. Microphytoplankton richness in Contas River, state of Bahia, northeastern Brazil. Checklist: Journal of Species Lists and Distribution 8(2): 218-223.

TELL, G.; CONFORTI, V. 1986. Euglenophyta Pigmentadas de la Argentina. Bibliotheca Phicologica 75: 1- 301. 